Devel opment of Fr equency Tunabl e, Medi um Power Gyr ot rons ( Gyr ot r on FU Ser i es) as Submi I I i met er Wave Radi at i on Sour ces

\begin{tabular}{|l|l|}
\hline 著者 & $\begin{array}{l}\text { I DEHARA Toshi t aka, OGAWA I sam, M TSUDO } \\
\text { Sei t ar o, PEREYASLAVETS M chael, N SH DA Naoki , } \\
\text { YOSH DA Ki yohi ko }\end{array}$ \\
\hline $\begin{array}{l}\text { j our nal or } \\
\text { publ i cat i on t i t l e }\end{array}$ & I EEE Tr ansact i ons on PI asma Sci ence \\
\hline vol une & PS 27 \\
\hline number & 2 \\
\hline page r ange & $340-354$ \\
\hline year & $1999-$ O4 \\
\hline URL & ht t p: //hdl . handl e. net /10098/1510 \\
\hline
\end{tabular}




\section{Development of Frequency Tunable, Medium Power Gyrotrons (Gyrotron FU Series) as Submillimeter Wave Radiation Sources}

Toshitaka Idehara, Isamu Ogawa, Seitaro Mitsudo, Michael Pereyaslavets, Naoki Nishida, and Kiyohiko Yoshida

(Invited Review)

\begin{abstract}
High frequency, medium power gyrotrons (Gyrotron FU series) have been developed at Fukui University as radiation sources covering a broadband from millimeter to submillimeter wave region. They have already been applied to high frequency electron spin resonance and to submillimeter wave scattering in plasma. Many parameters of the gorotron series could be useful for its applications in several new areas. The development and some applications of the Gyrotron FU series are summarized in this paper.
\end{abstract}

Index Terms-Gyrotron, submillimeter wave.

\section{INTRODUCTION}

T HE DEVELOPMENT of gyrotrons is proceeding in two directions. One is the development of high power, millimeter wave gyrotrons as the power sources for electron cyclotron heating of plasmas, electron cyclotron current drive of tokamaks [1]-[5], and for ceramic sintering [6], [7]. At present, 120-170 GHz, 1 MW, pulse gyrotrons are designed for plasma heating, and a $30 \mathrm{GHz}, 15 \mathrm{~kW}, \mathrm{CW}$ gyrotron for ceramic sintering.

The second direction is the development of high frequency, medium power gyrotrons [8], [9] as millimeter to submillimeter wave sources for plasma scattering measurements, [10], [11], electron spin resonance (ESR) experiments [12], and so on. Such gyrotrons operate on fundamentals, second, and even third harmonics of the electron cyclotron resonance under high magnetic field exceeding $10 \mathrm{~T}$. They have many advantages over other conventional sources, like frequency tunability and high power output.

Gyrotrons developed at Fukui University belong to the second group. They are high frequency, medium power gyrotrons covering a broad frequency band from millimeter to submillimeter wavelength region. Our "Gyrotron FU series" consists of eight gyrotrons. The latest gyrotron of the series, Gyrotron FU IVA [13] has achieved frequency tunability

Manuscript received July 20, 1998; revised January 19, 1999. This work was supported in part by a Grant-in-Aid from the Ministry of Education, Science. and Culture (Monbusho) of Japan, the International Joint Research Project from the Japan Society for the Promotion of Science, and the German-Japan from the Japan Society for the Promotion of Science, and the German-Japan
Scientific Research Program from Deutche Forschungsgemeinschaft (DFG) in Germany.

T. Idehara and S. Mitsudo are with the Research Center for the Development of Far-Infrared Region, Fukui University, Fukui 910-8507, Japan.

I. Ogawa, M. Pereyaslavets, N. Nishida, and K. Yoshida are with the Faculty of Engineering, Fukui University, Fukui 910-8507, Japan.

Publisher Item Identifier S 0093-3813(99)04857-2. from 160 to $889 \mathrm{GHz}$ with many single mode operations on fundamentals and on second harmonics of electron cyclotron frequency. This gyrotron is employed in high purity mode operation experiments. Its maximum frequency of $889 \mathrm{GHz}$ is the current record for high frequency operation of a gyrotron. The corresponding wavelength is $337 \mu \mathrm{m}$. This gyrotron is also applied as a radiation source in high frequency ESR experiments.

The previous gyrotron, Gyrotron FU IV [14], has also achieved frequency tunability up to $847 \mathrm{GHz}$. It was successfully employed in first experiments with frequency modulation [15] as well as for CW operation with high stability of amplitude and frequency [16].

Gyrotron FU III [17] achieved frequency tunability up to $636 \mathrm{GHz}$ operating on fundamentals, on second harmonics, and even on third harmonics. The gyrotron demonstrated amplitude modulation [18] and frequency step switching [19] of its output. It is useful for its applications as radiation source in infrared spectroscopy.

Gyrotron FU II [20] is the first submillimeter wave gyrotron in the series. Its maximum frequency is $402 \mathrm{GHz}$. It has been used for plasma scattering measurements on compact helical system (CHS) at the National Institute for Fusion Science (NIFS) in Japan. Its output power of about $100 \mathrm{~W}$ at $352 \mathrm{GHz}$ is transmitted by a circular waveguide system. It is later converted into a quasi-Gaussian mode and injected into the CHS plasma. A scattered signal is analyzed by a homodyne detection system. The measurement confirmed the excitation of a low frequency drift wave during neutral beam injection (NBI) heating. Such information is important to achieve the best plasma confinement. We intend to apply this gyrotron for plasma diagnostics on experimento tokamak esferico (ETE) at Instituto Nacional de Pesquisas Espaciais (INPE) in Brazil. The gyrotron was also used for studies of mode competition [21] and mode cooperation [22]. These studies give some insights for high efficient, single mode operations on second harmonics. The first gyrotron, Gyrotron FU I [23] is frequency tunable from 38-220 GHz. It bas been used as a radiation source for ESR experiment. Gyrotron FU IA has been applied as a radiation source for millimeter wave scattering measurement of WT-3 at Kyoto University [24].

Now we have constructed a new gyrotron, Gyrotron FU $\mathrm{V}$ [25] using a helium-free, $8 \mathrm{~T}$ superconducting magnet. The tube is demountable, because we will try to optimize all

0093-3813/99\$10.00 1999 IEEE 
TABLE I

Present Status of Gyrotron FU Sermes

\begin{tabular}{l|l|l}
\hline Name of gyrotron & Frequency range & Items which each gyrotron has achieved \\
\hline Gyrotron FU I & $38-220 \mathrm{GHz}$ & Radiation source for ESR experiment \\
\hline Gyrotron FU E & $90-300 \mathrm{GHz}$ & Radiation source for ESR experiment \\
\hline Gyrotron FU IA & $38-215 \mathrm{GHz}$ & $\begin{array}{l}\text { Radiation source for plasma scattering measurement of WT-3 } \\
\text { Studies on mode competition and mode cooperation }\end{array}$ \\
\hline Gyrotron FU II & $70-402 \mathrm{GHz}$ & Radiation source for plasma scattering measurement of CHS \\
\hline Gyrotron FU III & $100-636 \mathrm{GHz}$ & $\begin{array}{l}\text { 3rd harmonic operation in single modes, Amplitude } \\
\text { modulation, Frequency step switching }\end{array}$ \\
\hline Gyrotron FU IV & $160-847 \mathrm{GHz}$ & $\begin{array}{l}\text { Frequency modulation, cw operation for high stabilities of } \\
\text { amplitude and frequency }\end{array}$ \\
\hline Gyrotron FU IVA & $160-889 \mathrm{GHz}$ & $\begin{array}{l}\text { Higher frequency operalions by 3rd harmonics, High } \\
\text { purity mode operation, Radiation source for ESR experiment }\end{array}$ \\
\hline
\end{tabular}

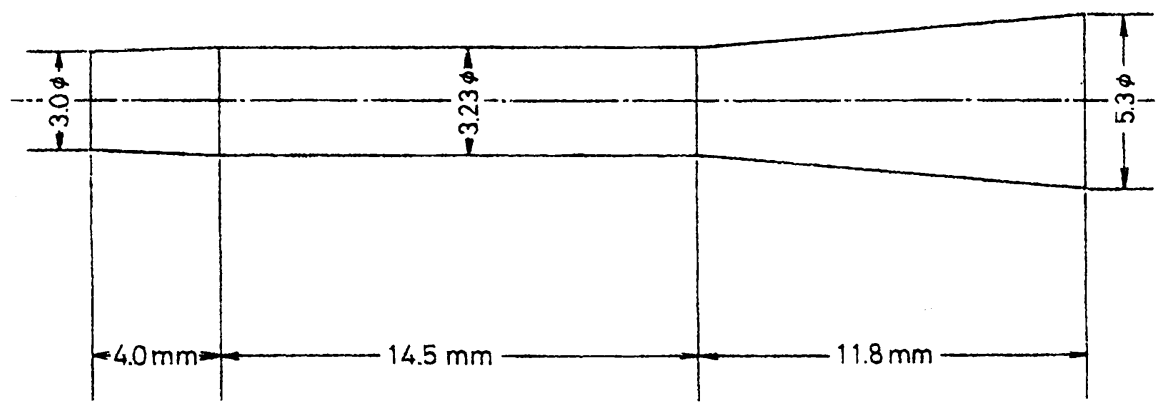

Fig. 1. The shape of a cylindrical cavity installed in Gyrotron FU IV.

components including the cavity, the transmission waveguide, the window, and so on. Our aim is to realize high mode purity operations. The gyrotron has the other advantage, namely, a long run in complete CW operation, because it is not restricted by the supply of liquid helium. After testing of its operation quality, the gyrotron will be applied for research in material physics, in plasma scattering phenomena, and so on.

The parameters achieved by Gyrotron FU series are summarized in this paper.

\section{DEVELOPMENT OF GYROTRON FU SERIES}

\section{A. Designs and Constructions of High Frequency, Harmonic Gyrotrons}

1) The Present Status of Gyrotron FU Series: Gyrotron FU series inciudes eight gyrotrons. Each gyrotron consists of a sealed-off gyrotron tube and a superconducting magnet, except Gyrotron FU II, which has a demountable tube. Table I summarizes the present status of gyrotrons included in Gyrotron FU series.

The design was carried out by computer simulations. We are using narrow cavities to get a good mode separation, and then to operate the gyrotrons in many single modes on fundamentals, second, even third harmonics. Such operation is important for our high frequency, harmonic gyrotrons. For example, the diameter of the previous gyrotron, Gyrotron, FU IV [14] is only $3.23 \mathrm{~mm}$, as is shown in Fig. 1. Beam currents sustaining $100 \mathrm{~W}$ output power are plotted in Fig. 2 for many cavity modes as functions of the magnetic field intensity $B_{0}$. The parameter $\left(N=f / f_{c}, f\right.$, and $f_{c}$ are the operating frequency and the electron cyclotron frequency) indicates the harmonic number. A $17 \mathrm{~T}$ superconducting magnet is used for Gyrotron FU IV. As it is obvious from the figure, there are many candidates for single mode operation on fundamentals $(N=1)$, second harmpnics $(N=2)$, and even on third harmonics $(N=3)$ in the region of field intensity from 14.5 to $17 \mathrm{~T}$. If we adjust field intensity $B_{0}$ to its optimal value, we can operate some of second harmonics in Fig. 2, suppressing mode competition with any fundamentals of higher output power. We can expect that the maximal frequency of the second harmonic is about $850 \mathrm{GHz}$ for single mode operation at $\mathrm{TE}_{4,8,1}$ cavity mode. The other gyrotrons in Gyrotron FU series are designed along the same lines as Gyrotron FU IV.

2) Gyrotron FU IVA: A schematic drawing of the latest gyrotron, Gyrotron FU IVA [13], is shown in Fig. 3. The gyrotron consists of a $17 \mathrm{~T}$ superconducting magnet and a sealed-off tube. A triode magnetron injection gun operates in the magnetic field leaking from the superconducting magnet. The mirror ratio, that is, the ratio of the main field intensity at the cavity region to the intensity at the cathode of the electron gun, is about 45 . Fig. 4 shows a computer simulation 


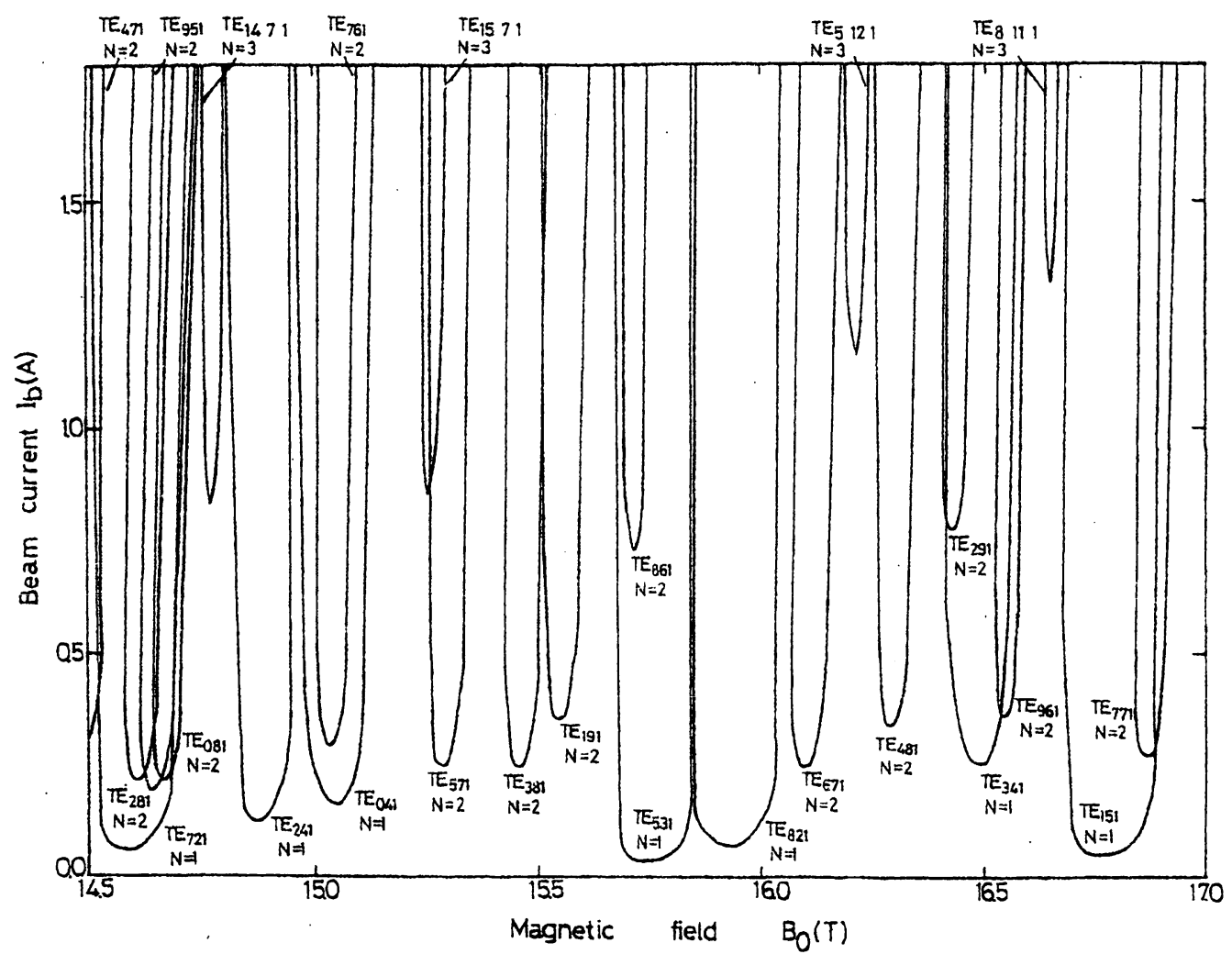

Fig. 2. Computer simulation results for beam currents $I_{b}$ sustaining $100 \mathrm{~W}$ outpur power for several cavity modes as functions of magnetic field intensity $B_{0}$. The parameter $N$ shows harmonic number.

result for anode voltage $V_{a}$ of the gun keeping the velocity ratio $\alpha$ at $1.5\left(\alpha=v_{1} / v_{2}, v_{1}\right.$, and $v_{2}$ are the perpendicular and parallel velocity components, respectively), as a function of field intensity $B_{0}$. Solid circles and open circles present results for Gyrotrons FU. IVA and IV, respectively. The solid circle encircled by an open circle corresponds to the second harmonic operation on $\mathrm{TE}_{1,9,1}$ cavity mode with frequency of $874 \mathrm{GHz}$. As it follows from this figure, we can control the pitch factor $\alpha$ in the broad range of field intensity from 5 to $17 \mathrm{~T}$, adjusting only the anode voitage $V_{a}$. The cavity with the diameter of $3 \mathrm{~mm}$ and the length of $6.25 \mathrm{~mm}$ only is installed in the gyrotron. The shape of the cavity is shown in Fig. 5. It contains a nonlinear up taper in the output region and smooth junctions to the cutoff down taper and to the output up taper to minimize mode conversion from the main cavity mode to competing modes. The cavity also has a small iris at the output end to increase its quality factor, and hence to decrease the starting current for working cavity modes. The design of this cavity was carried out very accurately by Wagner from Stuttgart University and Thumm from Forschungszentrum Karlsruhe [26]. Computer simulation results for conversion from the main cavity mode $\mathrm{TE}_{2,6,1}$ to its competing modes $\left(\mathrm{TE}_{2,5,1}, \mathrm{TE}_{2,7,1}\right.$, and so on) in the nonlinear up taper are shown in Fig. 6. A part of the main mode is converted to the $\mathrm{TE}_{2,5,1}$ and $\mathrm{TE}_{2,7,1}$ modes in the middle of the up-taper region, but almost all power returns back to the main mode at the end of the up taper. Calculation results predict that 99.3\% of total power can be transmitted through the up-taper region. Fig. 7 presents simulation results with equi-oscillationefficiency lines for third harmonic operation $(N=3)$ on the $\mathrm{TE}_{2,10,1}$ and $\mathrm{TE}_{9,7,1}$ modes, together with fundamental operation $(N=1)$ on the $\mathrm{TE}_{9,1,1}$ mode. The vertical and horizontal axes correspond to the injection point of electron beam in the cavity and field intensity $B_{0}$, respectively. The injection region of electrons in our device is shown. Single mode operation on third harmonic $(N=3)$ is possible only by adjusting field intensity $B_{0}$ to optimal conditions. The frequencies of $\mathrm{TE}_{2,10,1}$ and $\mathrm{TE}_{9,7,1}$ modes are 1022 and $1034 \mathrm{GHz}$. Therefore, we expect this gyrotron can achieve the breakthrough of 1 THz.

\section{B. Experimental Results for High Frequency, Harmonic Gyrotrons (Gyrotron FU Series)}

As it is shown in Table I, gyrotrons included in Gyrotron FU series are frequency step-tunable sources covering a wide wavelength range from millimeter to submillimeter wave region. The output powers are not so high, that is, from 


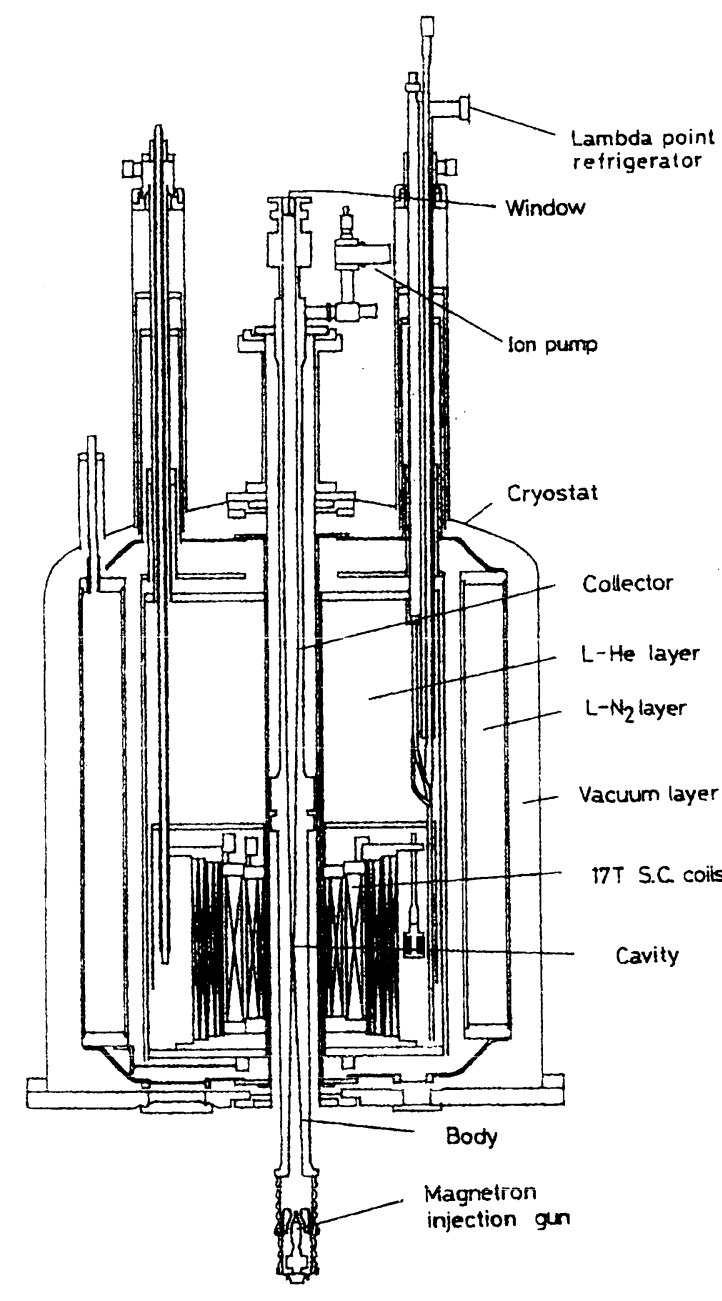

Fig. 3. A schematic drawing of Gyrotron FU IV A.

several hundreds watt to several tens kilowatt, for fundamental operation, and from several tens watt to several kilowant for second harmonic operation. The main results achieved up to now by Gyrotron FU series are summarized as following.

1) Frequency Tunability in Broadband from $38-889 \mathrm{GHz}$ [13], [14], [17], [20], [21]: Seven gyrotrons in the series have achieved frequency step tunability in the ranges shown in Table I. Fig. 8 shows a typical result of frequency measurement for Gyrotron FU IV using a Fabry-Perot interferometer. There are many instances of single mode operation on fundamentals [Fig. 8(a)] and on second harmonics [Fig. 8(b)]. Each interferometer pattern corresponds to field intensity $B_{0}$ indicated by an arrow. The measured wavelengths, their corresponding frequencies, and the cavity modes are shown in the figure. All frequencies achieved up to now by Gyrotron FU series are summarized as functions of field intensity $B_{0}$ in Fig. 9. Solid lines represent the fundamental $(N=1)$,

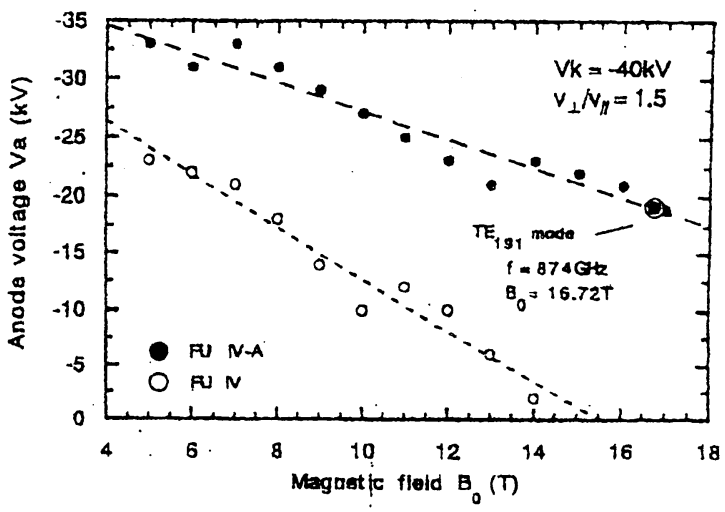

Fig. 4. Computer simulation results for anode voltage $V_{a}$ of electron gun keeping the velocity ratio $\alpha$ at 1.5 as functions of field intensity $B_{0} . \bullet$ and $\circ$ correspond to Gyrotrons FU IVA and FU IV.

the second, and the third harmonic $(N=2,3)$ resonances. Frequency step tunability from 38 to $889 \mathrm{GHz}$ is achieved for the fundamental, second, and third harmonic operation. Gyrotron FU IVA achieved the highest frequency of $889 \mathrm{GHz}$ for single mode operation on the $\mathrm{TE}_{8,6,1}$ cavity mode at the second harmonic $(N=2)$. The corresponding wavelength is $337 \mu \mathrm{m}$. Table II is a list of all frequencies achieved by Gyrotron FU IV, together with corresponding field intensities, cavity modes, and several output powers measured by a water load. In the case of Gyrotron FU IV, output powers are several hundreds watt at fundamentals and several tens watt at second harmonics. Similar frequency tunability has been achieved by each gyrotron of the series, as it is shown in Table $I$ and in Fig. 9. The Gyrotron FU series covers a broad frequency band from millimeter to submillimeter wave region $(38-889 \mathrm{GHz})$.

2) Amplitude Modulation [18], [27]: An amplitude modulation and frequency modulation of submillimeter wave gyrotrons are useful for their applications in various new areas as radiation sources. Gyrotron FU III has achieved amplitude modulation of its output. A modulation of the anode voltage $V_{a}$ will modulate the velocity distribution function of beam electrons, which, in its turn, will modulate the gyrotron output. Fig. 10 shows a typical result of amplitude modulation in the submillimeter wave gyrotron, with the modulation level of anode voltage $V_{a}$ expressed as the ratio $\Delta V_{a} / V_{a 0}$ as a parameter. The gyrotron, in this instance, is operating on the fundamental of the cyclotron frequency. The cavity mode is $\mathrm{TE}_{6,2,1}$, the frequency is $349 \mathrm{GHz}$, and the output power is about $60 \mathrm{~W}$. The modulating frequency is $5 \mathrm{kHz}$ and modulation mode is square wave. The upper traces show the high voltage pulse applied to the anode. The small square wave modulation signal is visible on the traces. The lower traces show the output power of the gyrotron. The modulation rate $\Delta P_{\text {out }} / P_{\text {uut }}$ of gyrotron output increases with the modulation rate $\Delta V_{a} / V_{a}$ of the anode voltage. The $100 \%$ modulation of the output $\left(\Delta P_{\text {out }} / P_{\text {out }}=1.0\right)$ is attained, when $\Delta V_{a} / V_{a}$ is only several percent $\left(\Delta V_{a} / V_{a} \sim 0.076\right)$. Fig. 11 shows the modulation rate $\Delta P_{\text {out }} / P_{\text {out }}$ of output power as functions 


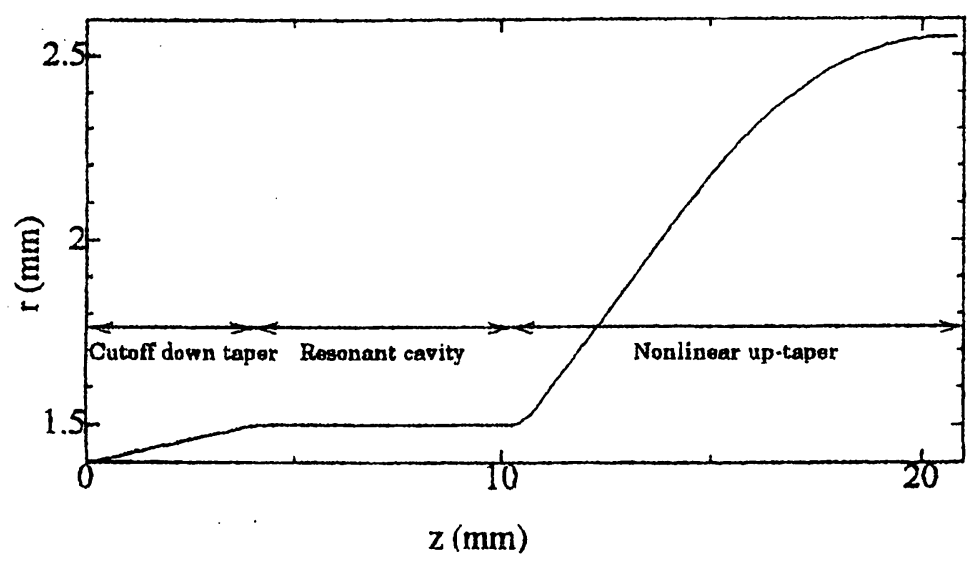

Fig. 5. The shape of the cavity installed in Gyrotron FU IVA.

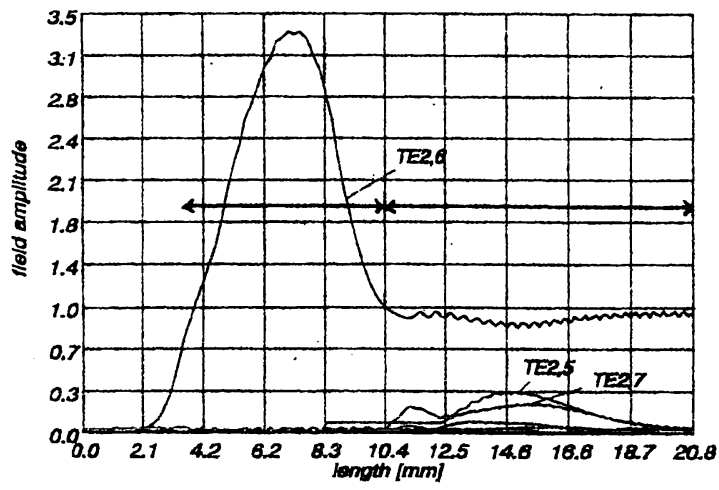

Fig. 6. Computer simulation result for mode conversions from the main cavity mode $\mathrm{TE}_{2,6,1}$ to its competing modes $\mathrm{TE}_{2,5,1}, \mathrm{TE}_{2,7,1}$, and so on, occurring in the nonlinear up-taper region.

of the modulation rate $\Delta V_{a} / V_{a 0}$, with the beam current as a parameter. $\Delta P_{\text {out }} / P_{\text {out }}$ is linearly proportional to $\Delta V_{a} / V_{a 0}$. This means the sinusoidal modulation of output power is possible by the sinusoidal modulation of anode voltage. Sinusoidal modulation of $P_{\text {out }}$ at the modulation frequency up to $600 \mathrm{kHz}$ has been achieved with the low $\Delta V_{a} / V_{a 0}$ value of $1.1 \times 10^{-3}$.

3) High Purity Mode Operations [13]: Gyrotron FU IVA is designed for high purity mode operations as described in the previous section. Carefully designed cavity, possessing a nonlinear up taper in the output side and smoothly connecting parts in the beginning of the cutoff taper and the nonlinear up taper, is installed. Such structure of the cavity will minimize the mode conversions from the main cavity mode to its competing modes [26].

The propagation angle of each mode in a cylindrical waveguide has its own value. (A different mode has a different angle.) We set a step-cut waveguide on the top of the gyrotron tube as shown in Fig. 12 and measured the angle dependency of emitted power by using a Vlasov type antenna. This measurement determines the power distribution for each mode.

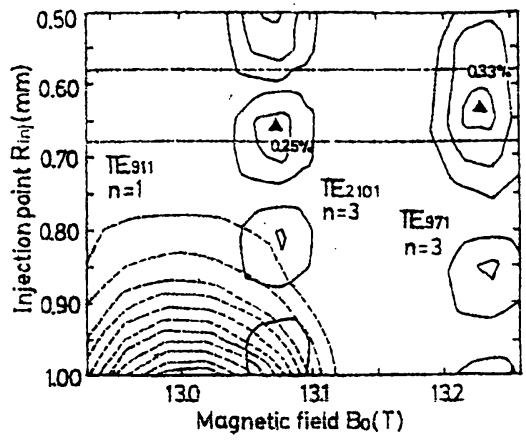

Fig. 7. Computer simulation result with equi-oscillation-efficiency lines for thind harmonic operation on $\mathrm{TB}_{2,10,1}$ and $\mathrm{TE}_{9,7,1}$ modes, together with fundamental operation on $\mathrm{TE}_{9,1,1}$ mode.

Table III summarizes the results for the main cavity mode $\mathrm{TE}_{031}$, whose resonant frequency is $323.8 \mathrm{GHz}$. In this case, a Fabry-Perot interferometer pattern shows the operation is single mode at the fundamental cyclotron resonance. The observed frequency determined from the interferometer pattern is $323 \mathrm{GHz}$, which is close to calculated value. Operation conditions are as follows: the acceleration voltage of electron beam $V_{b}=35 \mathrm{kV}$, the electron beam current $I_{b}=0.85 \mathrm{~A}$, and the field intensity in the cavity region $B_{0}=11.97 \mathrm{~T}$. In the measurement, $82 \%$ of the total power is emitted as the main cavity mode $\mathrm{TE}_{031}$.

In the table, calculation results for the complete tube [26] are also indicated on the right-hand side column. It shows that about $89.6 \%$ of the total power can penetrate from the cavity to the output window. Agreement between experimental and calculation results is fairly good. In Gyrotron FU IVA, the design of the cavity is complete, but the other part is not optimized. So, the total mode purity, which determines the mode purity of the output power, was degraded by ten or twenty percent.

4) Complete CW Operation with High Stability of Amplitude and Frequency [16]: Fig. 13 shows the variations of tempera- 


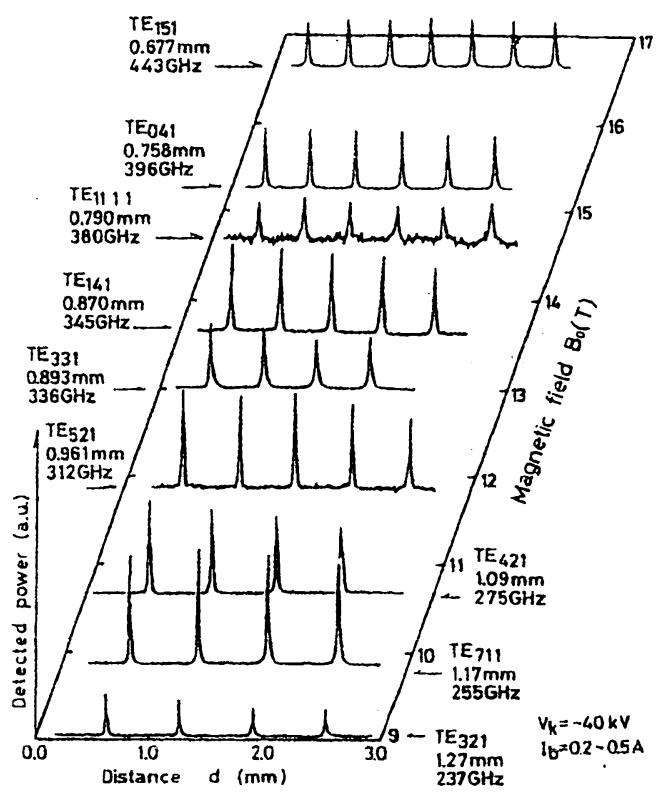

(a)

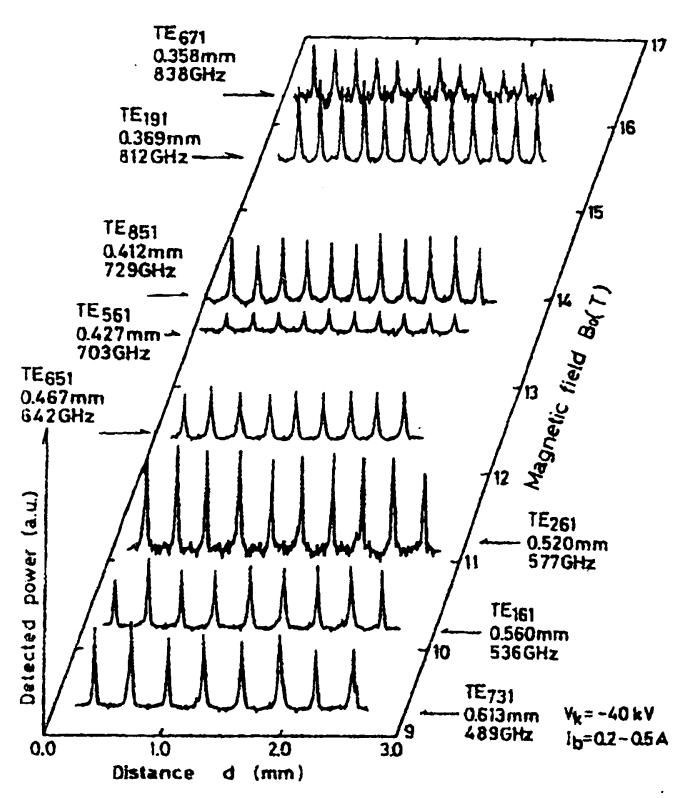

(b)

Fig. 8. A typical result of frequency measurement for Gyrotron FU IV using a Fabry-Perot interferometer. Interferometer patterns for. (a) the fundamentals and (b) the second harmonics.

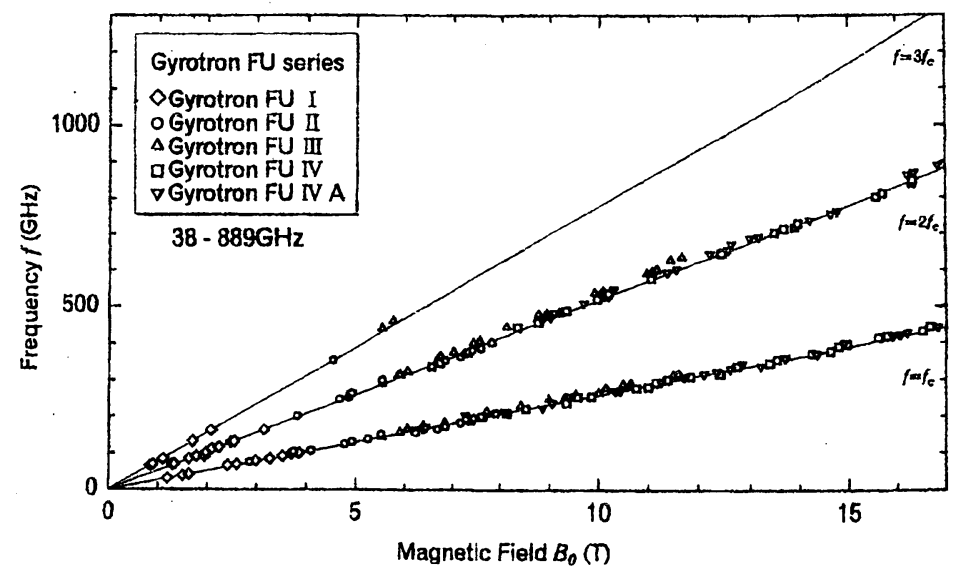

Fig. 9. All frequencies achieved up to now by Gyrotron FU series as functions of field intensity $B_{0}$. Solid curves show the fundamental, the second harmonic, and the third harmonic resonances.

ture of the water in the water load measured for several cavity modes by a thermocoupler as functions of time. The slope of each observed line is proportional to the corresponding output power. The results of power estimations are following: 4.5 $\mathrm{W}$ for $\mathrm{TE}_{021}$ mode at the frequency of $207.5 \mathrm{GHz}, 20.1 \mathrm{~W}$ for $\mathrm{TE}_{321}$ mode at $238.3 \mathrm{GHz}$, and $17.1 \mathrm{~W}$ for $\mathrm{TE}_{031}$ mode at $301.9 \mathrm{GHz}$. The longest period of the operation was five hours, which means "complete CW."

Similar measurements have been made for many other cavity modes. Table IV summarizes the results, together with field intensity in the cavity region $B_{0}$, accelerating voltage of elec- tron beam $V_{b}$, beam current $I_{b}$, cavity mode $T E_{m n 1}$, frequency $f$ measured by the Fabry-Perot interferometer and/or the modulation domain analyzer, input power $P_{\text {in }}$, and operation efficiency $\eta$.

The electron beam current $I_{b}$ is several tens of milliamperes. It was limited by the water cooling capacity. It is close to the starting currents of corresponding cavity modes. Therefore, operation is not so stable, but the powers obtained are moderately high and the efficiency $\eta$ exceeds $1 \%$ for a few modes.

One of the advantages of complete CW operation is stabilization of the frequency and the amplitude of the gyrotron 
TABLE II

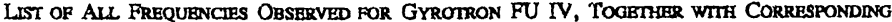

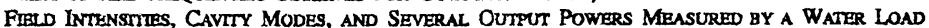

\begin{tabular}{|c|c|c|c|c|c|}
\hline $\begin{array}{l}B a \\
\text { (I) }\end{array}$ & $\begin{array}{l}f=f . \\
\left(G i x^{\prime}\right)\end{array}$ & $\begin{array}{l}f=2 f \\
(G+1 / 2)\end{array}$ & $\begin{array}{c}P \\
(W)\end{array}$ & m & $\mathbf{E}$ \\
\hline 623 & 159 & & \multirow{12}{*}{403} & 1 & 2 \\
\hline 6.55 & & 336 & & 3 & 3 \\
\hline 6.73 & & 346 & & 1 & 4 \\
\hline 7.31 & - & 376 & & 4 & 3 \\
\hline 7.38 & 190 & & & 5 & 1 \\
\hline 7.64 & 199 & - & & 2 & 2 \\
\hline 8.13 & 208 & \multirow{3}{*}{443} & & 0 & 2 \\
\hline 8.34 & & & & 3 & 4 \\
\hline 8.52 & 221 & & & 6 & 1 \\
\hline 8.77 & & 455 & & 9 & 2 \\
\hline 9.27 & - & 486 & & 0 & 5 \\
\hline 9.34 & & 489 & & 7 & 3 \\
\hline 9.35 & 237 & & \multirow[t]{3}{*}{456} & 3 & 2 \\
\hline 9.58 & 253 & & & 1 & 3 \\
\hline 9.86 & 255 & & & 7 & 1 \\
\hline 9.97 & & 521 & \multirow[t]{3}{*}{ - } & 11 & 2 \\
\hline 10.14 & & 529 & & 3 & 5 \\
\hline 10.20 & & 536 & & 1 & 6 \\
\hline 10.76 & 278 & \multirow{5}{*}{577} & \multirow[t]{5}{*}{253} & 4 & 2 \\
\hline 11.00 & 281 & & & 8 & 1 \\
\hline 11.05 & & & & 2 & 6 \\
\hline 11.15 & 293 & & & 2 & 3 \\
\hline $1: .38$ & 301 & & & 0 & 3 \\
\hline
\end{tabular}

\begin{tabular}{|c|c|c|c|c|c|}
\hline $\begin{array}{l}B 0 \\
\text { (I) }\end{array}$ & $\begin{array}{l}f=f e \\
\left(G f_{2}\right)\end{array}$ & $\begin{array}{l}f=2 f e \\
(G \in z)\end{array}$ & (iin) & $\begin{array}{l}\mathrm{T} \\
\mathrm{m}\end{array}$ & 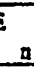 \\
\hline 11.85 & 309 & & & 5 & 2 \\
\hline 12.42 & 316 & & & 9 & 1 \\
\hline 1244 & & 647 & & 6 & 5 \\
\hline 12.74 & 337 & & 107 & 3 & 3 \\
\hline $13: 40$ & 346 & & & 1 & 4 \\
\hline 13.49 & & 703 & & 5 & 6 \\
\hline 13.55 & 356 & & 35 & 6 & 2 \\
\hline 13.68 & & 714 & & 3 & 7 \\
\hline 13.90 & & 718 & 51 & 1 & 8 \\
\hline 13.96 & & 729 & & 8 & 5 \\
\hline 14.25 & 372 & & 107 & 4 & 3 \\
\hline 14.63 & 378 & & & 11 & 1 \\
\hline 14.79 & 390 & & & 2 & 4 \\
\hline 14.97 & 396 & & & 0 & 4 \\
\hline 15.57 & & 802 & & 3 & 8 \\
\hline 15.62 & 414 & & & 5 & 3 \\
\hline 15.71 & & 812 & 63 & 1 & 9 \\
\hline 15.80 & 417 & & & 8 & 2 \\
\hline 16.30 & & 838 & & 6 & 7 \\
\hline 16.32 & & 847 & & 4 & 8 \\
\hline 16.52 & 431 & & & 3 & 4 \\
\hline 16.67 & 443 & & & 1 & 3 \\
\hline
\end{tabular}

a)

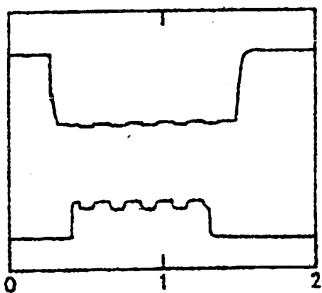

(a)

$\Delta P / P_{\text {out }}=1.5 \times 10^{-1}$ $\Delta V_{a} / V_{\text {an }}=1.1 \times 10^{-2}$

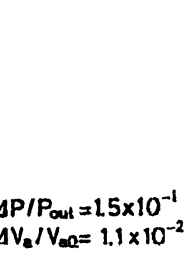

b)

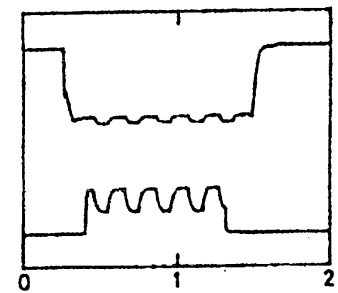

(b)

c)

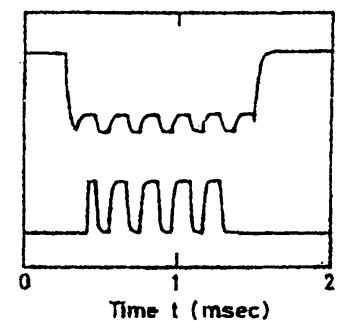

$\triangle P\left(P_{\text {out }}=10\right.$

$\Delta V_{a} / V_{a 0}=7.6 \times 10^{-2}$

(c)

Fig. 10. Amplitude modulation result for Gyrotron FU III. Upper traces are high voltage pulses applied to the anode and lower traces output powers of the gyrotron. Operation conditions: $\mathrm{TE}_{6,2,1}$ mode at the fundamental, modulation frequency is $5 \mathrm{kHz}$, and modulation mode is square wave, the frequency $f=349 \mathrm{GHz}$, unmodulated beam voltage $V_{b}=40 \mathrm{kV}$, and beam current $I_{b}=0.3 \mathrm{~A}$

output. We employ a current-stabilized high voltage power supply in our experiment to ensure stable operation. A variation in the output frequency was measured by a time-resolved frequency measurement system.

Fig. 14 shows a typical result of frequency measurement, together with the accelerating voltage $V_{b}$. The operation con- ditions are following: the field intensity in the cavity region $B_{0}=10.88 \mathrm{~T}$, the accelerating voltage of electron beam $V_{b}=14 \cdot \mathrm{kV}$, the beam current $I_{b}=80 \mathrm{~mA}$, the cavity mode $\mathrm{TE}_{031}$, the frequency $f=301.9 \mathrm{GHz}$, the input power $P_{\text {in }}=1.12 \mathrm{~kW}$, the output power $P_{\text {out }}=17.1 \mathrm{~W}$, and the operation efficiency $\eta=1.53 \%$. The measured frequency 


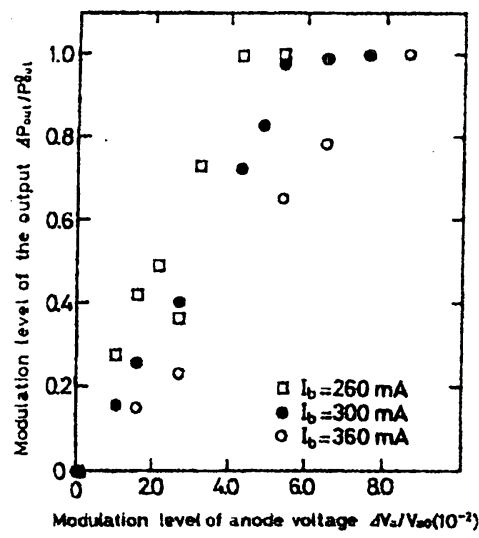

Fig. 11. Modulation of the ourput power $\Delta P_{\text {ouf }} / P_{\text {ont }}$ as functions of modulation level $\Delta V_{a} / V_{a}$, with beam current $I_{b}$ as a parameter.

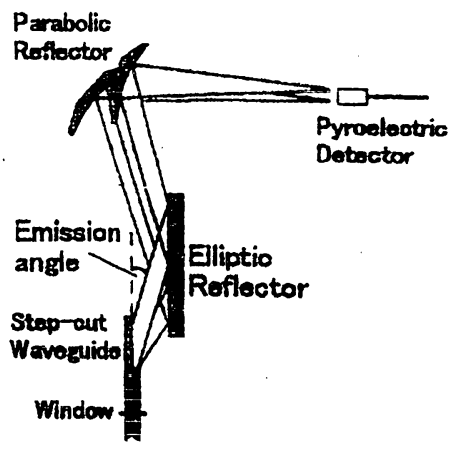

Fig. 12. The arrangement of a step-cut waveguide on the top of the gyrotron and quasi-optical Vlasov antenna for measurement of content ratio of each mode.

TABLE III

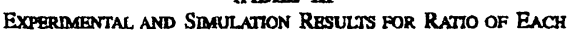
OUtPUT MODE, WHEN the CAVTTY OpERates ON TE 031 MODB

\begin{tabular}{|c|c|c|c|c|}
\hline $\begin{array}{l}\text { Cavity } \\
\text { mode }\end{array}$ & $\begin{array}{l}\text { Radiation angle } \\
a \text { (Degree) }\end{array}$ & $\begin{array}{c}\text { Detoced power } \\
\zeta(\mathrm{A})\end{array}$ & $\begin{array}{l}\text { Mode purity (\%) } \\
\text { Exporimemal }\end{array}$ & $\begin{array}{l}\text { Mode purity (\%) } \\
\text { Calculuted }\end{array}$ \\
\hline 01 & 3.23 & 0.00 & 0 & 0.02807 \\
\hline 2 & 5.93 & 0.30 & 1 & 1.59100 \\
\hline 3 & 8.62 & 38,41 & 82 & 895.5727 \\
\hline 4 & 11.33 & 6.62 & 14 & 2.28597 \\
\hline 05 & 14.05 & 1.32 & 3 & 0.02247 \\
\hline 06 & 16.81 & 0.00 & 0 & 0.00648 \\
\hline
\end{tabular}

variation $\delta f$ during $100 \mathrm{~ms}$ is $\delta f=4.1 \mathrm{MHz}$. The variation ratio $\delta f / f=1.3 \times 10^{-5}$. The frequency variation is increased, probably due to a variation in the accelerating voltage $V_{b}$. The variation amplitude of $V_{b}$ in the figure is about $10 \mathrm{~V}$. It causes a small variation in the electron mass, and from it we can estimate the variation in the electron cyclotron frequency $\delta f_{c}$ as $5.6 \mathrm{MHz}$. Its variation ratio $\delta f_{c} / f_{c}=1.9 \times 10^{-5}$. The experimentally obtained values of $\delta f / f$ are in good agreement with the estimated value of $\delta f_{c} / f_{c}$.

Similar measurements bave been carried out for several other modes. The resuits are summarized in Table V. In the

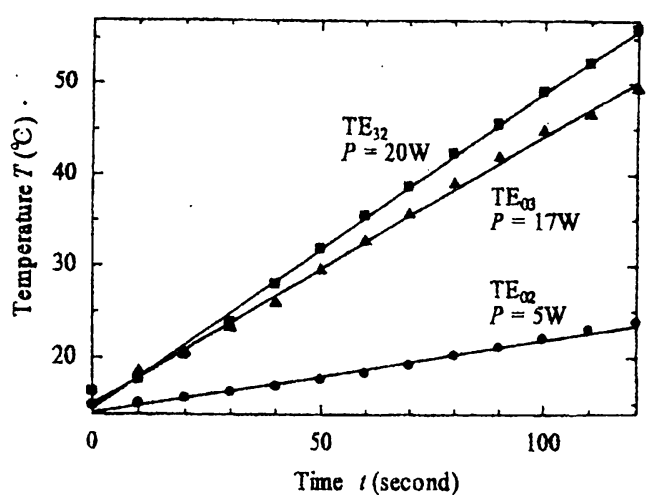

Fig. 13. The results of power measurements using a water load

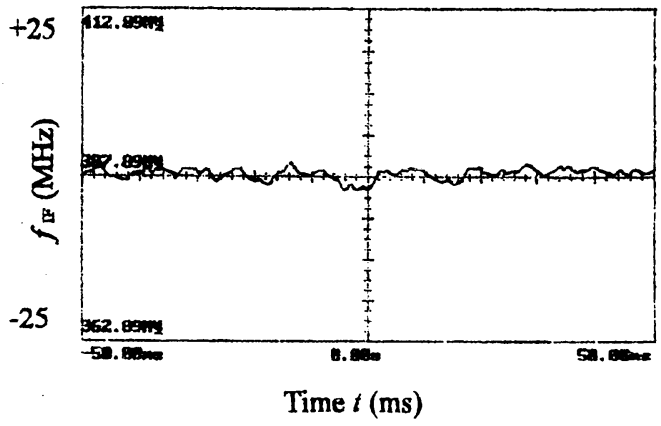

(a)

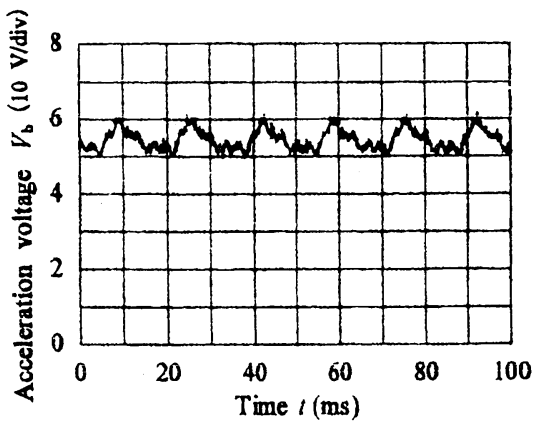

(b)

Fig. 14. (a) A result of frequency measurement. A full scale in the vertical axis is $50 \mathrm{MHz}$ and (b) a simultaneous observation result of acceleration voltage $V_{b} .10 \mathrm{~V} / \mathrm{div}$ in the vertical axis.

table, the frequency variation $\delta f$ during $100 \mathrm{~ms}$ is listed for several cavity modes, together with the cavity mode $\mathrm{TE}_{m n 1}$, the frequency of the local oscillator $f_{L}$, the harmonic number of the mixer $n$, the frequency $f_{\mathrm{IF}}$ detected by the modulation domain analyzer, and the determined operation frequency $f$, as well as calculated resonant frequency of the cavity. For all cases, $\delta f$ is several $\mathrm{MHz}$ and $\delta f / f$ is of the order of $10^{-5}$.

The stability of the operation frequency may be improved by further stabilization of the output voltage of a high voltage power supply. 
TABLE IV

The Results of Measurements of Output Power $P_{\text {out, }}$, Together with Find Intensity in the Cavity Region $B_{0}$, accel eration Voltage op Electron Beam $V_{b}$, Beam Current $I_{b}$, Cavity Mode TE $m n$, Frequency $f$ Mrasured by a FABRY-PEROT INTERFEROMETER AND/OR a HETEROdYNR DETECTION SYSTEM,. INPUT POWER $P_{\text {In }}$, AND OPERATION EFICTENCY

\begin{tabular}{c|c|c|c|c|c|c|c}
\hline $\begin{array}{c}B_{0} \\
(\mathrm{~T})\end{array}$ & $\begin{array}{c}V_{\mathrm{b}} \\
(\mathrm{kV})\end{array}$ & $\begin{array}{c}I_{\mathrm{b}} \\
(\mathrm{mA})\end{array}$ & $\mathrm{TE}$ & $\begin{array}{c}f \\
(\mathrm{GHz})\end{array}$ & $\begin{array}{c}P_{\mathrm{m}} \\
(\mathrm{W})\end{array}$ & $\begin{array}{c}P_{\text {out }} \\
(\mathrm{W})\end{array}$ & $\begin{array}{c}\eta \\
(\%)\end{array}$ \\
\hline 7.09 & 12 & 64 & 22 & 199.3 & 768 & & \\
\hline 7.53 & 12 & 32 & 02 & 207.5 & 384 & 4.5 & 1.2 \\
\hline 7.95 & 14 & 32 & 61 & 221 & 448 & 2.0 & 0.45 \\
\hline 8.52 & 14 & 68 & 32 & 238.3 & 952 & 20.1 & 2.1 \\
\hline 9.06 & 14 & 75 & 13 & 252 & 1050 & 9.8 & 0.93 \\
\hline 9.14 & 14 & 67 & 71 & 253.6 & 938 & 3.2 & 0.34 \\
\hline 10.53 & 15 & 80 & 23 & 295 & 1200 & 11.6 & 0.97 \\
\hline 10.88 & 14 & 80 & 03 & 301.9 & 1120 & 17.1 & 1.53 \\
\hline
\end{tabular}

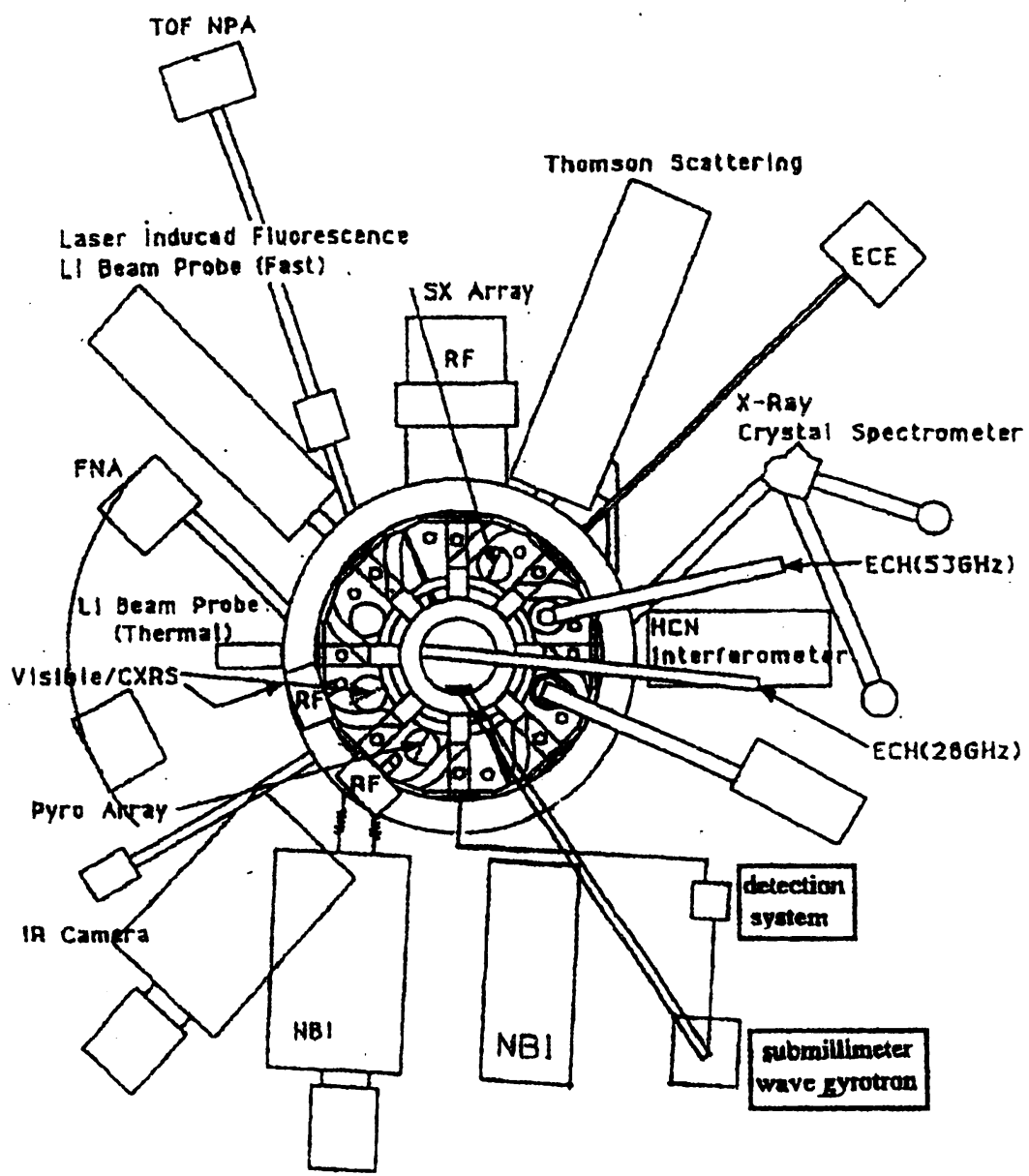

Fig. 15. Schematic drawing of installation of scattering measurement system using Gyrotron FU II on CHS. 


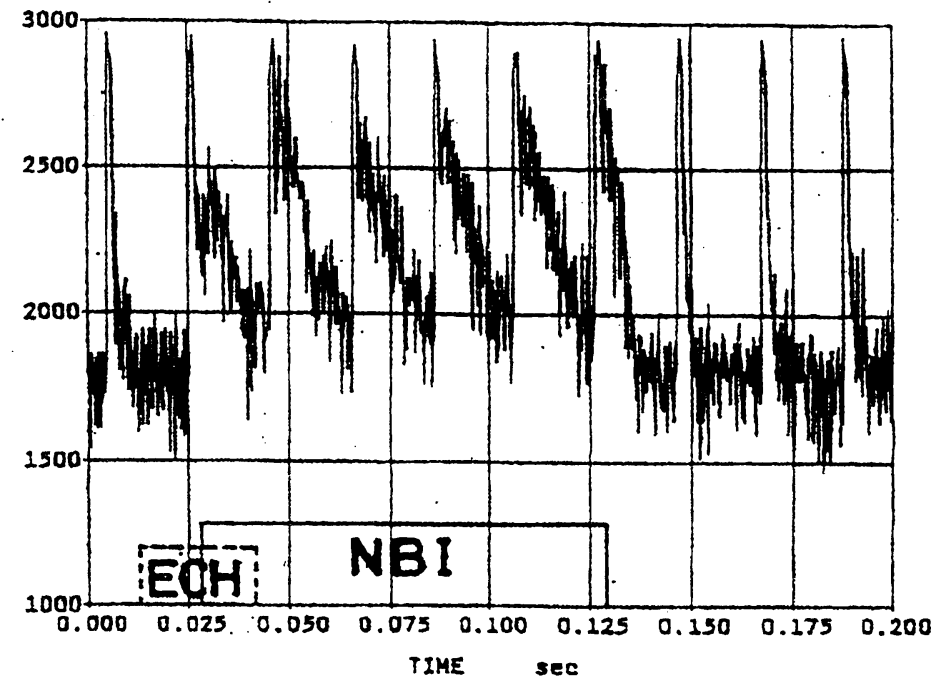

Fig. 16. Time evolution of frequency spectrum of scattered signal. Time intervals of ECH and NBI pulses are indicated.

TABLE V

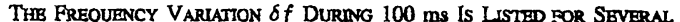
CAVTTY MODES, TOGETHER WITH CAVITY MODB TE mn1, FRBQUENCY OP LOCAL OSCDIATOR $f_{L}$. HARMONIC NUMBER OP THE MEXBR $n$, FRBQURENCY $f_{\text {TF }}$ Detrected by the Modulation Domain ANalyzzR, Detersanted Oferration

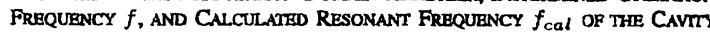

\begin{tabular}{|c|c|c|c|c|c|c|}
\hline Mode & $\begin{array}{l}\text { Local } \\
\text { Oscillator } \\
f_{L} \text { (Grtz) }\end{array}$ & $\begin{array}{c}\text { Harmoxic } \\
\text { Number } \\
\text { n }\end{array}$ & $\begin{array}{l}\text { Modulation } \\
\text { Domain } \\
\text { Amalyzer } \\
f_{\mathrm{p}}(\mathrm{MHz})\end{array}$ & $\begin{array}{c}f \\
\text { meas. } \\
\text { (GHz) }\end{array}$ & $\underset{(M F f z)}{\delta f}$ & $\underset{\text { cal }}{f}$ \\
\hline $\mathrm{TE}_{2}$ & 16.572 & 12 & 429 & 199.293 & 3.4 & 198.395 \\
\hline $\mathrm{IE}_{2}$ & 12.227 & 17 & 342 & 207.517 & 4.4 & 207.526 \\
\hline$\pi E_{n}$ & 13.246 & 18 & 168 & 238.260 & 2.6 & 237.027 \\
\hline$T E_{n}$ & 10.548 & 24 & 483 & 253.630 & 5.2 & 253.634 \\
\hline
\end{tabular}

\section{APPLICATION OF GYROTRON FU SERIES}

\section{A. Application to Plasma Scattering Measurement}

As it is shown in Table I, Gyrotron FU II is employed as a radiation source for plasma scattering measurements on the compact helical system (CHS) of the National Institute for Fusion Science (NIFS). A gyrotron is a well-suited source for plasma scattering measurement, because of its high power possibilities in submillimeter wave range. Gyrouron FU II delivers long pulses (pulse width is changed up to $0.6 \mathrm{~s}$ ) of moderately high power $(\sim 110 \mathrm{~W})$ at submillimeter wavelengths $(f \sim 354$ $\mathrm{GHz}, \lambda=0.847 \mathrm{~mm}$ ) [10]. Its application enables us to improve the $S / N$ ratio of a measurement and to observe low level density fluctuations.

Fig. 15 shows the installation of a scattering measurement system on CHS. The gyrotron output is transmitted by conventional over-sized circular waveguides and converted to a two-dimensionally focused, linearly polarized, quasiGaussian beam, before it is injected into plasma. Scattered wave from plasma is received by hom antennas installed in the plasma vessel. It is converted into low frequency signal by a homodyne detection system.
The scattering measurements with scattering angles of 4.4 and $8.8^{\circ}$ are carried out in NBI heated plasma and in "ion cyclotron resonance of frequency (ICRF)" heated plasmas. Fig. 16 shows a typical result of scattering measurement with the angle of $8.8^{\circ}$ for NBI heated plasma. The trace shows one frequency spectrum of the scattered signal after another. Each spectrum covers the frequency band from 0 to $1 \mathrm{MHz}$ and was obtained in $20 \mathrm{~ms}$. The plasma is produced by a microwave pulse for electron cyclotron heating (ECH) and heated by a NBI puise. As it is obvious, some broaciening of the spectrum occurs during NBI heating. After the NBI pulse, the broadening cannot be observed. It is detected up to about $600 \mathrm{kHz}$, and is possibly due to scattering by drift waves excited spontaneously in the NBI heating phase.

Fig. 17 shows: a) a scattering measurement result for ICRF heated plasma, together with b) the corresponding reflection measurement using a reflectmeter. The plasma is fired at the time of $10 \mathrm{~ms}$ by an ECH puise, and an ICRF heating pulse is applied in the time interval from $40-90 \mathrm{~ms}$. Each curve shows time evolution of scattered waye power for respective frequency interval. The scattering angle of $8.8^{\circ}$ corresponds to wavenumber of $11.4 \mathrm{~cm}^{-1}$. The increase in scattered wave power is observed during the ICRF heating. The results of the reflection measurement are in reasonable agreement with the scattering measurement and support the availability of both measurements.

Observed scattered signals suggest that some instability connected with the drift wave occurs during NBI or ICRF heating. This phenomenon will be dangerous for a good plasma confinement. We will continue the measurement under various plasma parameters to study the effect of this instability on plasma confinement.

\section{B. Application to ESR Experiment [12], [28]}

Gyrotrons FU I, FU E, and FU IVA are employed as radiation sources for ESR spectroscopy. The gyrotrons as 


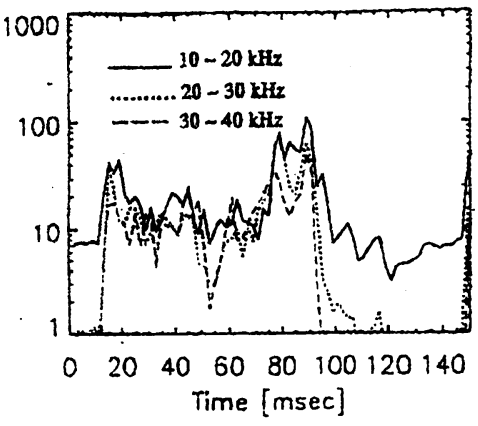

(a)

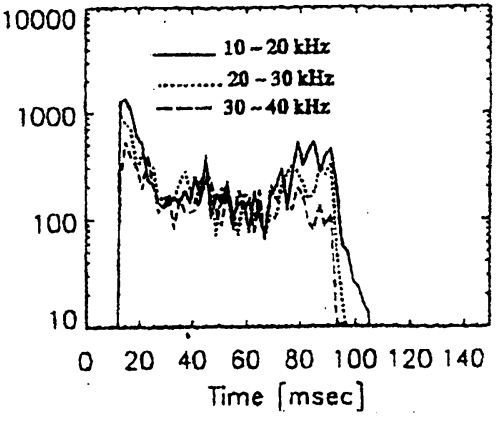

(b)

Fig. 17. (a) Time evolution of scattered wave power for respective frequency interval and (b) time evolution of reflected wave power for respective froquency interval.

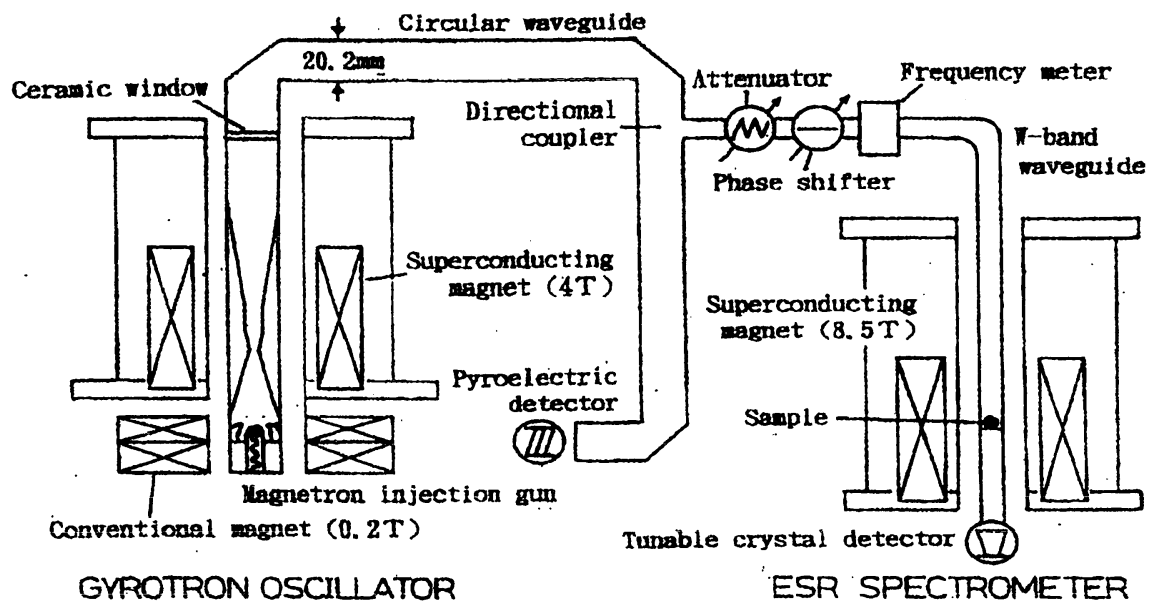

Fig. 18. A block diagram of ESR spectrometer using Gyrotron FU I as a radiation source.

millimeter to submillimeter wave sources have important advantages, compared to other conventional sources; for example, frequency tunability in broad band and moderately high output power. Because of such advantages, our gyrotions are useful for ESR spectroscopy in the millimeter to submillimeter wavelength range to study nonlinear phenomena of ESR.

Fig. 18 is a block diagram of an ESR spectrometer using Gyrotron FU I as a radiation source. The output power of the gyrotron is transmitted by an oversized circular waveguide and drives the spectrometer. The main part of the spectrometer is constructed using a W-band waveguide system. The superconducting magnet generates high magnetic field up to $8.5 \mathrm{~T}$ at the sample holder. The transmitted power is detected by a crystal detector.

Fig. 19 shows typical patterns demonstrating ESR resonant absorption for a standard sample, $\alpha, \alpha$-diphenyl- $\beta$-picryl hydrazyl (DPPH). For a single crystal sample [Fig. 19(a)], a sharp resonance is observed. The half value width $\Delta H$ is equal to $2 \times 10^{-4} \mathrm{~T}$. The value is comparable with the theoretical prediction. On the other hand, for polycrystalline powder sample, the resonance is broadened, because of an anisotropy of $g$ value.

The measured resonance magnetic field $H_{0}$ is plotted in Fig. 20 as a function of the frequency $\nu$. The solid line corresponds to the theoretical result. The ESR spectrometer using Gyrotron FU I as a radiation source covers a broad frequency band in millimeter wave region.

Fig. 21 shows observed half value widths of the resonance lines as functions of frequency $\nu$. Hollow circles are the results for single crystal sample and solid circles for polycrystalline powder sample. Solid lines show theoretical predictions taking into account an anisotropy of $g$ values. They are in good agreement with experimental results.

As another example, $\mathrm{ESR}$ of $\mathrm{Cr}^{3+}$ in dark ruby is measured using Gyrotron FU IVA as a radiation power source. Fig. 22 shows a typical ruby ESR spectrum obtained by sweeping the magnetic field. Absorption signals are observed at three differ. ent magnetic fields corresponding to fine structure transitions of the spin state interval ${ }^{4} \mathrm{~A}$ ground state of the $\mathrm{Cr}^{3+}$. Fine structure spectral lines for three transitions are observed on the 

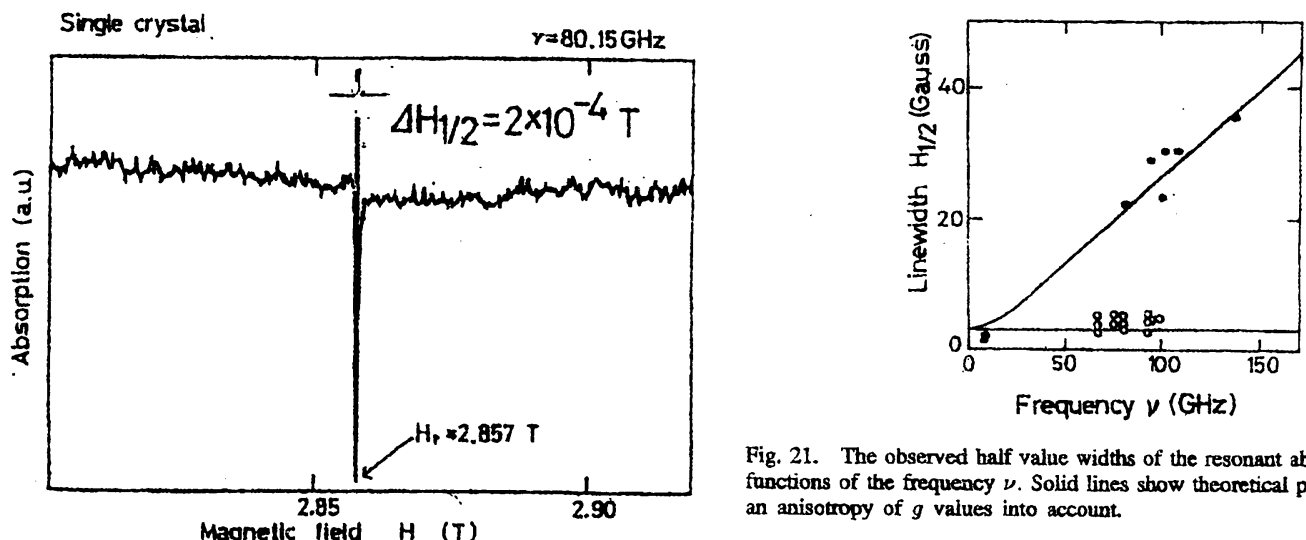

Fig. 21. The observed half value widths of the resonant absorption lines as functions of the frequency $\nu$. Solid lines show theoretical predictions mating an anisotropy of $g$ values into account.

(a)

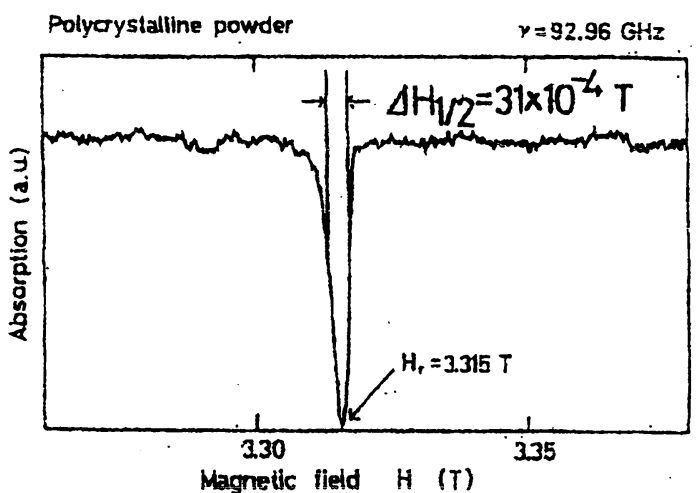

(b)

Fig. 19. ESR resonant absorption patterns for standand samples DPPH: (a) for a single crystal sample and (b) for polycrystalline powder sample.

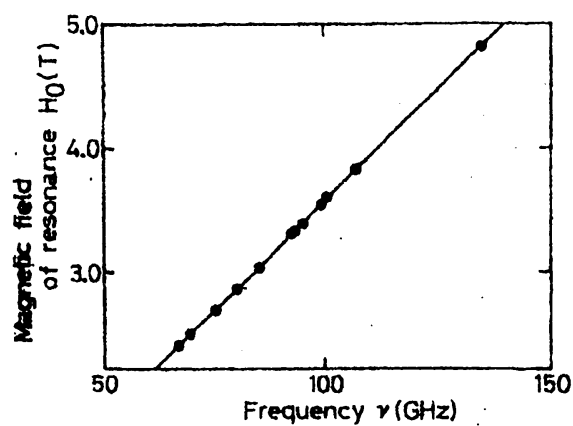

Fig. 20. The observed resonance fields $H_{0}$ as a function of the frequency $\nu$.

spectrum. The two ESR signals around $3.2 \mathrm{~T}$ are for a DPPH (on the low magnetic field side) and for ruby (for the transition between $\mid+1 / 2>$ and $\mid-1 / 2>$ ), respectively. The resonant magnetic fields depend on the orientation of the $c$ axis.

The field intensities $H_{0}$ corresponding to the resonant absorptions are plotted in Fig. 23 as functions of the angle

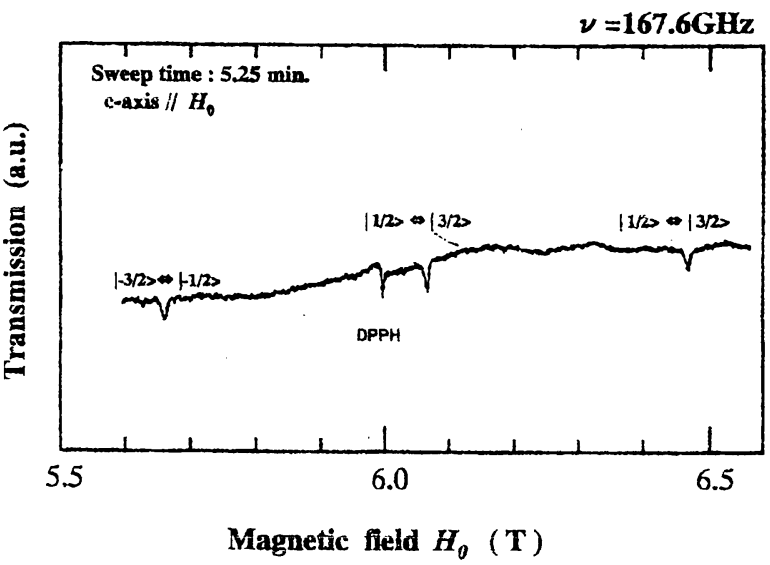

Fig. 22. ESR spectrum of $\mathrm{Cr}^{3+}\left({ }^{4} \mathrm{~A}\right)$ in ruby as a function of magnetic fieid $H_{0}$ at $\nu=167.62 \mathrm{GHz}$. ESR signal around 6.0T is DPPH's one as a correct marker of the field.

$\theta$ between the directions of the magnetic field and the $c$ axis of ruby. Solid curves are calculation results, where $g$ factors, and the fine structure constant $D$ are determined for the best fit with the experimental results, as follows:

$$
g_{\|}=1.9803, \quad g_{\perp}=1.9813, \text { and } D=-5.742 \mathrm{GHz} \text {. }
$$

Figs. 24 and 25 show the frequency dependence of the $g$ factors and the fine structure constants, respectively. Solid curves are calculation results using a formula including $\nu^{2}$ terms. It seems that the experimental results are in good agreement with the calculation results. ESR studies at highex frequency help to understand the variations in $g$ factors and in the $D$ value.

We will try the ESR experiment in a near future in a broad frequency band in submillimeter wave region. We are now preparing a pulse magnet for a submillimeter wave ESR spectrometer. Its intensity can rise up to about $30 \mathrm{~T}$.

\section{SUMMARY}

Gyrotron FU series in Fukui University consists of seven gyrotrons. Each of them is designed to get many single mode operations on fundamentals, second, and even third harmonics 


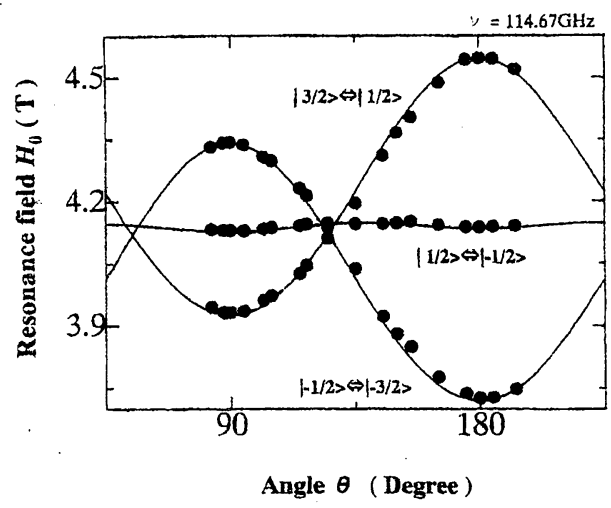

Fig. 23. The angular dependence of the three ESR transition lines of $\mathrm{Cr}^{3+}\left({ }^{+} \mathrm{A}\right)$ in ruby. Solid lines are calculation results by third order perturbation on the Price Hamiltonian.

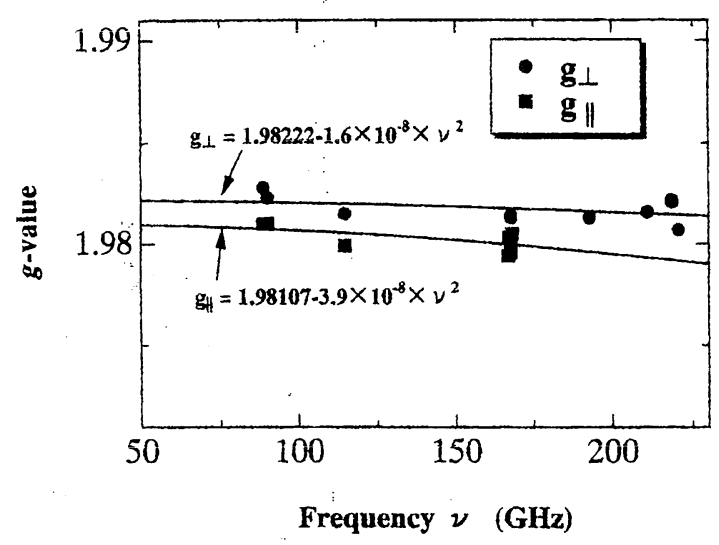

Fig. 24. $g$ values of $\mathrm{Cr}^{3+}\left({ }^{4} \mathrm{~A}\right)$ in ruby versus the resonance frequency.

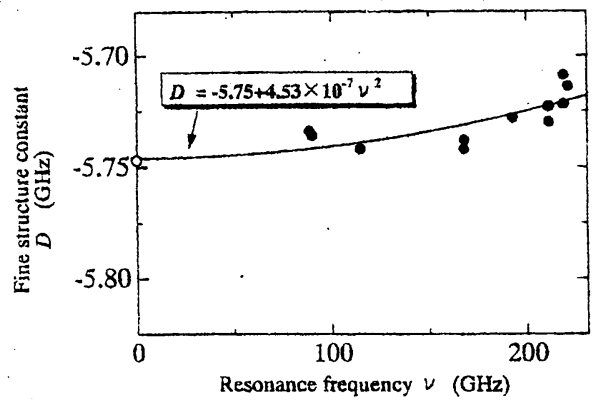

Fig. 25. The fine structure constant $D$ of $\mathrm{Cr}^{3+}\left({ }^{4} \mathrm{~A}\right)$ in ruby versus the resonance frequency. Solid lines are calculation results, supposing that the frequency dependence of $D$ is proportional to $\nu^{2}$.

of electron cyclotron resonance. Such a design results in high frequency, medium power gyrotrons covering broad frequency band from millimeter to submillimeter wave region. The results achieved by our gyrotrons are summarized as follows.

1) These gyrotrons have achieved frequency tunability from $38-889 \mathrm{GHz}$.
2) The output powers are moderately high; that is, from several hundreds watt to several tens kilowatt for fundamental operation and from several tens watt to several kilowatt for second harmonic operation.

3) Higher harmonic operation is demonstrated up to the fourth harmonic. This is necessary for development of high frequency gyrotrons covering the whole submillimeter wavelength range.

4) Mode competition and mode cooperation between the fundamental and the second harmonic are studied in detail. A mode competition is not convenient for our high frequency, harmonic gyrotron. Therefore, it should be avoided.

5) Amplitude modulation, frequency step switching, and frequency modulation of gyrotron output were achieved for its application in various new areas.

6) Complete CW operation has been performed with high stability of amplitude and frequency of the gyrotron output.

Gyrotron FU II has already been employed for plasma scattering measurements on CHS in NIFS, and Gyrotron FU I, FU E, and FU IVA for ESR experiments to study the resonance line widths of DPPH and frequency dependencies of $g$ factors and a fine structure constant $D$ of ruby.

At present, the newest gyrotron Gyrotron FU V has just been designed and constructed. The gyrotron consists of a heliumfree $8 \mathrm{~T}$ superconducting magnet and a demountable tube. It should achieve time-unlimited, high quality mode operation for applications in far infrared spectroscopy.

\section{ACKNOWLEDGMENT}

The authors wish to thank Dr. G. F. Brand from the University of Sydney, Prof. M. Thumm from Forschungszentrum Karlsruhe, and Dr. W. Kasparek and Dr. D. Wagner from Universitat Stuttgart for their invaluable discussions.

\section{REFERENCES}

[1] B. Piosczyk, O. Braz, G. Dammertz, C. T. Iatrou, S. Kern, M. Kuntze, G. Michel, A. Mobius, and M. Thumm, "Operation of a coaxial gyrotron with a dual RF-beam output," in Dig. 22nd Int. Conf. Infrared Millimeter Woves, H. E. Freund, Ed Wintergreen, VA, July 1997, pp. 114-115.

[2] K. Sakamoto, A. Kasugai, M. Tsuneoka, K. Takahashi, Yu. Mkeda, T. Imai, T. Kariya, K. Hayashi, Y. Mitsunaka, Y. Hirata, Y. Ito, and Y. Okazaki, "Development of high power $170 \mathrm{GHz}$ gyrotron for ITER," in Dig. 22nd Int. Conf. Infrared Millimeter Waves, H. E. Freund, Ed. Wintergreen, VA, July 1997 , pp. 106-107.

(3) V. E. Myasnikov, M. V. Agapova, V. V. Alikaev, A. A. Bogdashov, A. A. Borschegovsky, G. G. Denisov, V. A. Flyagin, A. Sh. Fix, V. 1. Ilyin, V. N. Ilyin, V. A. Khmara, D. V. Khmara, A. N. Kostyna, V. O. Nichiporenko, L. G. Popov, and V. E. Zapevalov, "Long-puise operation of 110-GHz 1-MW gyrotron," in Dig. 22nd Int. Conf. Infrared Millimeter Waves, H. E. Freund, Ed. Wintergreen, VA. July 1997, pp. $102-103$.

[4] K. Feich, P. Borchard. C. Cahalan, T. S. Chu, H. Jory, C. M. Loring, and C. P. Moeller, "Status of $1 \mathrm{MW}$ CW gyrotron development at CPI," in Dig. 21 st Int. Conf. Infrared Millimeter Waves, M. von Ontenberg and H. U. Mueller, Eds. Humboldt University zu Berlin, Bertin, Germany, July 1996.

[5] T. Kimura, J: P. Hogge, R. Advani, D. Denison, K. E. Kreischer, and R. J. Temkin, "Investigation of megawatt power level gyrotrons for ITER," in Dig. 2 Ist Int. Conf. Infrared Millimeter Waves, M. von Ortenberg and H. U. Mueller, Eds. Humboldt University zu Berlin, Berlin, Germany, July 1996. 
[6] I. I. Antakov, I. G. Gachev, V. I. Kurbatov, E. V. Sokolov, E. A. Solujanova, and E. V. Zasypkin, "A Ka-band $10 \mathrm{~kW}$ CW efficient compact gyrotron for materials processing," in Dig. 21 st "Ins. Conf. Infrared Millimeter Waves, M. von Ontenberg and H. U. Mueller, Eds. Humbold University za Berlin, Berlin, Germany, July 1996.

[7] H. Asano, T. Kikunaga, K. Memmi. F. Sato, and T.Tsukamoto, "A $28 \mathrm{GHz}$ gyrotron with a permanent magnet system for industry applications," in Dig. 21st Int. Conf. Infrared Millimeter Waves, M. von Ontenberg and H. U. Mueller, Eds. Humboldt University zu Berlin, Berlin, Germany, July 1996

[8] T. Idehara, T. Tatsukawa, I. Ogawa, Y. Shimizu, N. Nishida, and K. Yoshida, "Development and applications of submillimeter wave gyrotrons," in Proc. 3nd Int. Conf. Strong Microwave Plasmas, Moscow, Russia, 1997, vol. 2, pp. 634-659.

[9] K. D. Hong, G. F. Brand, and T. Idehara, "A $150-600 \mathrm{GHz}$ step-tunable gyrotron," J. Appl. Phys., vol. 74, nc. 8, pp. 5250-5258, 1993

[10] I. Ogawa, K. Yoshisue, H. Ibe, T. Idehara, and K. Kawahata, "Longpulse operation of a submillimeter wave gyrotron and its application to plasma scattering measurement," Rev. Sci. Instrum., vol. 65, no. 10, pp. 1788-1789, 1994

[11] P. W. Fekete, G. F. Brand, and T. Idehara, "Scattering from discrete Alfven waves in a tokamak using a gyrotron radiation source," Plasma Phys. Controlled Fusion, vol. 36, no. 9, pp. 1407-1417, 1994.

[12] T. Tatsukawa, T. Maeda, H. Sasai, T. Idehara, M. Mekata, T. Saito, and $T$. Kanemaki, "ESR spectroscopy with a wide frequency range using a gyrotion as a radiation power source," Int. J. Infrared and Millimeter Waves, vol. 16, no. 2, pp. 293-305, 1995.

[13] T. Idehara, N. Nishida, K. Yushida, I. Ogawa, T. Tatsukawa, D. Wagner, G. Gantenbein, W. Kasparek, and M. Thumm, "High freguency and high mode purity operations of Gyrotron FU IVA," Int. J. Infrared and Millimeter Waves, vol. 19, no. 7, pp. 919-930, 1998

[14] T. Idehara, Y. Shimizu, K. Ichikawa, S. Makino, K. Shibutani, T. Tatsukawa, I. Ogawa, Y. Okazaki, and T. Okamoto, "Development of a medium power, submillimeter wave gyrotron using a $17 \mathrm{~T}$ superconducting magnet," Phys. Plasmas, vol. 2, no. 9, pp. 3246-3248, 1995.

[15] T. Idehara, M. Pereyasiavets, N. Nishida, K. Yoshida, and I. Ogawa, "Frequency modulation in a submillimeter-wave gyrotron," Phys. Rev. Lett., vol. 31, no. 9, pp. 1973-1976, 1998.

[16] T. Idehara, K. Yoshida, N. Nishida, I. Ogawa, M. Pereyaslavets, and T. Tatsukawa, "CW operation of a submillimeter wave gyrotron (Gyrotron FU IV) for high stability of the output frequency," Int. J. Infrared Millimeter Waves, vol. 19, no. 6, pp. 793-801, 1998.

[17] Y. Shimizu, S. Makino, K. Ichikawa, T. Kanemaki, I Ogawa, Tatsukawa, and T. Idehara, "Development of a submillimeter wave gyrotron using a $12 \mathrm{~T}$ superconducting magnet," Phys. Plasmas, vol. 2, no. 6, pp. $2110-2116,1995$.

[18] T. Idehara, Y. Shimizu, S. Makino, K. Ichikawa, T. Tatsukawa, Ogawa, and G. F. Brand, "High-frequency, amplitude modulation of a submillimeter wave, medium power gyrotron," Phys. Plasma, vol. 1 no. 3, pp. $461-463,1994$.

[19] - "Rapid frequency step-switching of a submillimeter wave gyrotron by modulation of the electron beam." Phys. Plasma 1, vol. 1, no. 6. pp. 1774-1776, 1994

[20] T. Idehara, T. Tatsukawa, I. Ogawa, H. Tanabe, T. Mori, S. Wada, G. F. Brand, and M. H. Brennan, "Development of a second cyclotron harmonic gyrotron operating at sabmillimeter wavelengths," Phys. Fluids $B$, vol. 4, no. 1, pp. $267-273,1992$.

[21] T. Idehara, T. Tatsukawa, I. Ogawa, T. Mori, H. Tanabe, S. Wada, G F. Brand, and M. H. Brennan, "Competition between fundamental and second-harmonic operations in a submillimeter wave gymotron," Appl. Phys. Lett., vol. 58, no. 15, pp. 1594-1596, 1991.

[22] T. Idehara and Y. Shimizu, "Mode cooperation in a submillimeter wave gyrotron," Phys. Plasma, vol. 1, no. 10, pp. 3145-3147, 1994.

[23] T. Idehara, T. Tatsukawa, H. Tanabe, S. Matsumoto, K. Kunieda, K. Hemmi, and T. Kanemaki, "High frequency, step tunable, cyclotron harmonic gyrotron," Phys. Fluids B, yol. 3, no. 7, pp. 1766-1772, 1991

[24] Y. Terumichi, S. Kubo, A. Ando, Y. Yanagimoto, K. Ogura, H. Tanaka, J. Takabashi, I. Tonai, M. Nakamura, T. Maekawa, S. Tanaka, and T. Idehara, "Study on low frequency density fluctuations in the WT-2 tokamak by $\mathrm{mm}$ and submm scattering," in Dig. 9th Int. Conf. Infrared Millimeter Waves, K. Mizuno, Ed. Takarazuka, Japan, Oct. 22-26, 1984, pp. $411-412$.

[25] T. Idehara, 1. Ogawa, S. Mitsudo, M. L. Percyaslavets, T. Tsuchida, and M. Ui, "Development of a submillimeter wave gyrotron (Gyrotron FU V)," in Dig. 23th Int. Conf. Infrared Millimeter Waves, Essex, U.K., Sept. 1998.

[26] D. Wagner, M. Thumm, G. Gantenbein, W. Kasparek, and T. Idehara,
"Analysis of a complete gyrotron oscillator using the scattering matrix description," Int. J. Infrared Millimeter Waves, vol. 19, no. 2, pp.

[27] T. Idehara, Y. Shimizu, S. Makino, K. Ichikawa, T. Tatsukawa, I Ogawa, and G. F. Brand, "Amplitude modnlation of a submillimeter wave gyrotron output," Int. J. Infrared Millimeter Waves, vol. 18, no. 2. pp. 391-403, 1997.

[28] T. Tatsukawa, T. Shirai, T. Imaizumi, T. Idehara, I. Ogawa, and T. Kanemaki, "Ruby ESR over a wide frequency range in the millimeter wave region," Int. J. Infrared Millimeter Waves, vol. 19, no. 6, pp. $859-874,1998$

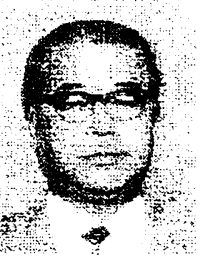

Toshitaka Idehara was bom in Ibara, Japan, on April 15,1940 . He received the B.S. degree in mathematics from Kyoto University, Japan, in 1963 and the M.S. and the D.S. degrees in physics from Kyoto University in 1965 and 1968 , respectively.

After graduating from Kyoto' University, he joined Fukai University, Japan, and was a Lecturer from 1968 to 1970 and an Associate Professor from 1970 to 1990 . During this term, he worked from 1970 to 1990 . During this tem, he worked
in fundamental plasma physics. After 1979 , his interest was directed toward the development of high frequency gyrotrons. From 1990 to 1999 , he was a Professor in the Applied Physics Department, Fukui University, and from 1992 to 1999 , he was Head of the Laboratory for Application of Superconducting Magnet. Fukui University. Since 1999, he has been a Professor and the Director of the Fukui University. Since 1999, he has been a Professor and the Director of the
Research Center for Development of Far-Infrared Region, Fukui University. His current research interests inchude development of frequency tunable submillimeter wave gyrotrons and their applications to plasma diagnostics and material physics.

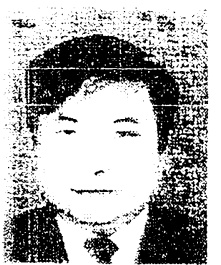

Isamu Ogawa was born in Hamada, Japan, on August 15, 1955. He received the B.S, and M.S. degrees in physics from Ehime University in 1979 and 1981, respectively. He received the D.S. degree in physics from Nagoya University in 1987.

After graduating from Nagoya University, he joined Fukui University, Japan. From 1989 to 1991 , he was a Research Associate. Since 1991, he has been an Associate Professor in the Cryogenic Lab oratory, Fukui University. His interest is directed to the system converting the output of a high frequency gyrotron into a Gaussian beam and its application to the plasma scattering measurement.

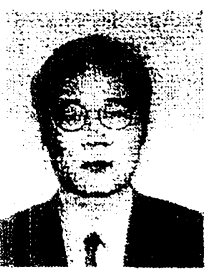

Seitaro Mitsudo was born in Okayama, Japan, on April 17, 1965. He received the B.S., the M.S. and the D.S. degrees in physics from Okayama University in 1989, 1991, and 1994, respectively.

After graduating from Okayama University, he joined the Institute for Materials Research of Tohoku University. From 1994 to 1998, he was a Research Associate. During this term, he worked in high field magnetism. From 1998 to 1999 , he was with the Applied Physics Department of Fukui University, Japan, as an Associate Professor. Since 1999, he has been an Associate Professor of the Research Center for Development of Far-Infrared Region, Fukui University. His current research interests include development of frequency nunable submillimeter wave gyrotrons and their applications for submillimeter wave ESR. 


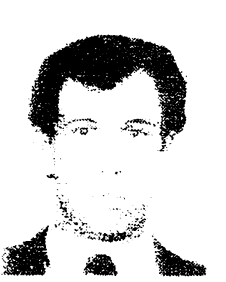

Michael Pereyasiavets received the Ph.D. degree in electrical engineering from the IRE of the Academy of Sciences.

He was previously with the Department of Electrical Engineering, Physical Electronics, at Tel-Aviv University, Israel. He has been a Visiting Scientist University, Israel. He has been a Visiting Scientist
at Fukui University, Japan, the National Institure for at Fukui University, Japan, the National Institute for sruhe, Germany. His research interests are in the area of microwave devices, field structure transformers, and mode converters.

Dr. Pereyaslavets holds research awands from the Japanese Society for the Promotion of Science (JSPS) and from MINERVA of the Max-Planck Sociery.

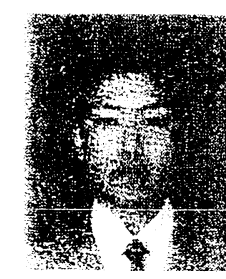

Naoki Nishida was born in Fukui, Japan, on April 30, 1973. He received the B.S. and the M.S. degrees in applied physics from Fukni University in 1996 and 1998 , respectively.

After graduating from Fukui University, he joined After graduating from Fukui University, he joined
Furukawa Electric Company as a Researcher. His research interests include the development of submillimeter wave gyrotrons.
Kiyohiko Yoshida was born in Fukui, Japan, on November 11, 1973. He received the B.S. and M.S. degrees in applied physics from Fukui University in 1996 and 1998, respectively.

After graduating from Fukui University, he joined After graduating from Fukui University, he joined
Mitsubishi Electric Company as a Researcher. His research interests include the development of submillimeter wave gyrotrons. 